\section{Radial Flow in the Cone-Plate Viscometer}

CONE-PLATE viscometers are widely used to-day to measure the flow properties of both Newtonian and non-Newtonian materials. The basic idea which led to the cone-plate geometry came from Mooney and Ewart ${ }^{1}$, who were trying to eliminate end corrections in coaxial cylinder viscometers. In deriving the equations governing cone-plate flow, Mooney and Ewart assumed that the sample moved in circular lamina only, with no radial component of velocity. This assumption has been generally accepted, and it still appears in a recent mathematical study of the cone-plate viscometer ${ }^{2}$. However, I have now directly observed a radial component of motion in cone-plate flow of both Newtonian and non-Newtonian materials.

One of the instruments used in this work was a Ferranti-Shirley cone-plate viscometer, which has been deseribed by McKennell ${ }^{3,4}$. The Ferranti instrument has four cones ranging from 2 to $8 \mathrm{~cm}$. in diameter, each having an angle of about $20^{\prime}$. The other viscometer was made in this laboratory, using transparent acrylic plastic for the cone and plate. The transparent cone is $10 \mathrm{~cm}$. in diameter, and the cone angle is $2^{\circ}$.

Newtonian materials examined were mineral oils ranging from 1 to 10 poises at $25^{\circ} \mathrm{C}$. and polyisobutene (U.S. National Bureau of Standards, Oil $O B$ ) of 219 poises at $25^{\circ} \mathrm{C}$. Experiments were performed by filling the cone-plate gap with oil and then placing a drop of the same oil, dyed red (Sudan red $4 B A$ ), at the outer edge of the gap. The cone was then rotated. In the case of the transparent viscometer, the coloured oil was seen to spread around the periphery of the cone. Then, with further turning, the coloured circular band spread inward towards the centre, maintaining a sharp boundary with the clear oil. About 1,000 cone revolutions were necessary to bring the dye boundary half-way to the centre of the cone. This experiment olearly demonstrates mass transfer in an inward radial diroction. Obviously thero must also be a balancing outward flow, but the path of the circulation is not yet known.

The rate of inward motion of the colour was far greater than can be obtained from diffusion (as determined by experiment). It also appeared to be independent of oil viscosity and of cone angular velocity, although these points need to be dotermined more carefully. Since the radial velocity was small relative to the cone angular velocity, the energy consumed in radial flow is probably very small, and the phenomenon does not in fact appear to affect the accuracy of viscosity determinations on Newtonian oils made with the Ferranti cone-plate viscometer.

In a further experiment, a drop of coloured oil was placed at the cone apex (that is, the circular centre of the cone-plate gap). When the cone was rotated, there was no radial migration of the colour. This suggests that there is an inner volume stagnant to radial circulation.

It should be noted that the usefulness of coloured oil as a flow tracer is very limited if the cone and plate are opaque, as in the Ferranti viscometer. The act of opening the cone-plate gap in order to observe the colour causes gross radial flow and distorts the result. On opening the gap of the transparent coneplate viscometer, it was soen that the oil was first drawn inward as the plate was lowered. Then, as the gap continued to increase, gravity broke the central column of oil which then flowed outward agnin. A ring of coloured oil survived the flow without turbulent mixing, but its location was not the samo as before the cone-plate gap was opened.

The non-Newtonian materials examined so far have been lubricating greases, which show complex flow properties such as yield values and time-dependent shear thinning. Experiments similar to those for the oils, using pigmented grease as the flow tracer, have demonstrated that there is also a radial component of cone-plate flow in greases. However, the phenomenon seems more complicated. It has been found that the stiffer a grease is, the more likely it is to flow radially outward and actually be ejected from the cone-plate gap. With a very stiff grease (National Lubricating Grease Institute Consistency Grade No. 4), most of the sample was extruded from the gap within a revolution or two of the cone. Soft greases were extruded to a lesser extent, if at all, and in some cases the escape of the sample from the gap seemed to depend critically on cone velocity. It is not clear yet whether the phenomenon of escape of grease is due to the same forces which act on Newtonian fluids or to some non-Newtonian characteristic such as normal forces.

These findings are preliminary, and they raise many questions. However, if one accepts these phenomena as real (that is, not caused by minor deviations from symmetry in the instrument), then two conclusions may be drawn. The first is that theoretical analysis of cone-plate flow needs to be extended to account for the radial flow component. The second is that the radial component may be an important factor in coneplate viscometry of non-Newtonian materials, even though it is not very important for Newtonian ones.

\section{David B. Cox}

Research Department,

Socony Mobil Oil Co., Inc.,

Paulsboro, New Jersey.

${ }^{1}$ Mooney, M., and Ewart, R. H., Physics, 5, 350 (1934).

2 Slattery, J. C., J. Colloid Sci., 16, 431 (1961).

${ }^{3}$ McKennell, R., Proc. Second Intern. Cong. Rheol., 350 (1953).

"McKennell, R., Anal. Chem., 28, 1710 (1956).

\section{CRYSTALLOGRAPHY}

\section{Whisker Growth from Quartz}

THE growth of whiskers from fused quartz has recently been reported by Jaccodine and Kline'. Whiskers have also been observed on single crystals of quartz prepared by the hydrothermal process ${ }^{2}$. $\mathrm{X}$-ray examination has now revealed the nature of these growths.

Two whiskers, about $17 \mu$ and $35 \mu$ respectively in diameter, obtained from the furnace process, were each found to be single crystals of high-cristobalite, with the whisker growth axis along [111]. The cubic lattice constant $a=6.99 \pm 0.02 \AA$., as compared with the literature valuc ${ }^{3}$ of $7.0459 \AA$. Precession photographs, with the whisker growth axis along the spindle axis, contained only sharp Bragg reflexions; more than a single reciprocal layer appeared for any givon orientation. Reflexions obtained on the rotation photographs taken about [111] were drawn out along the row lines through an angle of about $4^{\circ}$, indicating a lack of long-range order normal to this axis. Both whiskers were isotropic, viewed in polarized light.

A single whisker of about $50 \mu$ diameter, produced in the hydrothermal process, was identified as 